\title{
Mixed Doubles Match Technical and Tactical Analysis of World Badminton Champion Based on Mathematical Statistics
}

\author{
Bing Zhang, Feng Li, Weiqi Jiang \\ Institute of Physical Education, Huanggang Normal University, Huangzhou, China \\ Email: tiyuxi@qq.com
}

Received July $3^{\text {rd }}$, 2013; revised August $3^{\text {rd }}$, 2013; accepted August $10^{\text {th }}, 2013$

\begin{abstract}
Copyright (c) 2013 Bing Zhang et al. This is an open access article distributed under the Creative Commons Attribution License, which permits unrestricted use, distribution, and reproduction in any medium, provided the original work is properly cited.
\end{abstract}

\begin{abstract}
This paper uses mathematical statistics method and analyzes the main scoring techniques: the serve technique, the jump smash technique, the net lob technique, the pushing backcourt ball technique and the smash technique by analyzing the data of the 29th Olympic Games by He Hanbin/Yu Yang (China) and Nova/Nasir (Indonesia). The analysis shows that: in the badminton mixed doubles match, since both sides are partners for many years, in order to win the game you must have good badminton technique; particularly the error rate of the net lob technique and the pushing backcourt ball technique is high, which needs further practice. The study plays a certain role in promoting the development of badminton technique and the popularization of badminton sports.
\end{abstract}

Keywords: Echnical and Tactical Analysis; Badminton; Mathematical Statistics

\section{Introduction}

The most exciting moment in badminton competition is the smash technique in doubles, where one side jumps high and smashes in the backcourt, the other side hits the ball back to the backcourt. In doubles many scores often rely on smashing in the backcourt. In doubles one should avoid to be the defensive side (Tian, 2004). Moreover one needs offensive, which is the victory method in the doubles. The basic tactical intent in doubles is to make the opponent hit the ball up. When the opponents hit the ball upward, you can attack through the under pressure. When you must hit the ball up, you're in the defense. At this time, your consciousness should be looking for opportunities to counterattack. The most successful defense is to make opponents can no longer attack next shot (Zhang et al., 2013).

Many studies at home and abroad have shown that the adept skills and quick reactions are necessary in the doubles. But one needs more than this as good double players (Chen, 2009). The most critical consciousness is looking for opposite side's vulnerabilities and looking for the best counter attack opportunities in defense. The key to defensive is not to turn two people into wall, although hitting back the opponent's ball back can consume opponent's physical stamina. But successful defense should counterattack, which is the so-called active defense (Pan, 2001). When you strike the ball high, you must realize that if your ball has no threat, you may be probably in a defensive position. The offensive way is various. Similarly you also need to apply various techniques to obtain initiative according to opponent's technique and stance (Geng, 2004).

In this paper, we consult related information online and read some related badminton magazines. There are not many technical analyses for the badminton mixed doubles in the world. Thus it is necessary to conduct related technical analysis on live games of Chinese athletes He Hanbin/Yu Yang and the Indonesian athletes Nova/Nasir (Cheng, 2005). This paper uses mathematical statistics methods to analyze the technical and tactical of world badminton champion, who plays a certain role in promoting the development of badminton technique and the popularization of badminton sports.

\section{Research Object}

This article consulted competition technical and tactical data at the 29th Olympic Games of two sets of the world's top athletes He Hanbin/Yu Yang (China) and Nova/Nasir (Indonesia) through the Internet, as shown in Table 1.

\section{Analysis and Discussion}

\section{Winning and Losing Points Analysis}

On August 16, 2008 in Beijing Chinese players He Hanbin/ Yu Yang lost to the Indonesian athletes Nova/Nasir by 1:2 in the 29th Olympic Games badminton mixed game (Cheng, 2008). Chinese players win the first game by scoring 21 points, among which directly attack scored 8 points (accounting for $38.08 \%$ of the total score). The Indonesian players send 13 points due to mistakes (accounting for $61.88 \%$ of the total score). The Chinese players directly mistakes 6 points in the first game (accounting for $28.56 \%$ of the total losing score). The major mistakes: one pushing backcourt ball (accounting for $4.76 \%$ of the total losing score), one smash ball (accounting for $4.76 \%$ of the total losing score), 2 deep clear balls (accounting for $9.52 \%$ of the total losing score); Indonesian players score 11 points by direct attack (accounting for $73.32 \%$ of Chinese players' total losing points), 2 jump smash (accounting for $13.33 \%$ of Chinese players' total losing points), 2 pushing backcourt 
Table 1.

China and India semi-final technical data sheets of badminton mixed doubles in Beijing 2008 Olympic Games.

\begin{tabular}{|c|c|c|c|c|c|c|c|}
\hline Name & Scores & Gains and losses situation & Serve & Jump smash & Push backcourt ball & Smash & Total \\
\hline \multirow{11}{*}{ He Hanbin/Yu Yang } & \multirow{4}{*}{ The first game 21} & The success number & 2 & 4 & 2 & 0 & 8 \\
\hline & & Percentage & 9.52 & 19.04 & 9.52 & 0 & 38.08 \\
\hline & & The mistake number & 0 & 2 & 3 & 1 & 6 \\
\hline & & Percentage & 0 & 9.52 & 14.28 & 4.76 & 28.56 \\
\hline & \multirow{4}{*}{ The second game 11} & The success number & 1 & 2 & 1 & 2 & 6 \\
\hline & & Percentage & 9.09 & 18.18 & 9.09 & 18.18 & 54.54 \\
\hline & & The mistake number & 0 & 5 & 6 & 1 & 12 \\
\hline & & Percentage & 0 & 23.70 & 28.56 & 4.76 & 57.12 \\
\hline & \multirow{4}{*}{ The third game 21} & The success number & 0 & 0 & 2 & 4 & 6 \\
\hline & & Percentage & 0 & 0 & 9.52 & 38.08 & 47.6 \\
\hline & & The mistake number & 1 & 5 & 5 & 2 & 13 \\
\hline \multirow{12}{*}{ Nova/Nasir } & & Percentage & 4.34 & 21.70 & 21.70 & 8.68 & 56.42 \\
\hline & \multirow{4}{*}{ The first game 15} & The success number & 0 & 4 & 4 & 3 & 11 \\
\hline & & Percentage & 0 & 26.66 & 26.66 & 20.00 & 73.32 \\
\hline & & The mistake number & 7 & 0 & 6 & 0 & 13 \\
\hline & & Percentage & 33.33 & 0 & 28.56 & 0 & 61.88 \\
\hline & \multirow{4}{*}{ The second game 21} & The success number & 2 & 3 & 3 & 1 & 9 \\
\hline & & Percentage & 9.52 & 14.28 & 14.28 & 4.76 & 42.84 \\
\hline & & The mistake number & 1 & 2 & 1 & 1 & 5 \\
\hline & & Percentage & 8.33 & 16.66 & 8.33 & 8.33 & 41.65 \\
\hline & \multirow{3}{*}{ The third game 23} & The success number & 0 & 3 & 4 & 3 & 10 \\
\hline & & Percentage & 0 & 13.02 & 17.36 & 13.02 & 43.40 \\
\hline & & The mistake number & 1 & 6 & 7 & 1 & 15 \\
\hline
\end{tabular}

ball (accounting for $13.33 \%$ of Chinese players' total losing points), 3 smash balls (accounting for $20.00 \%$ of Chinese players' total losing points).

Chinese players lost the second game by 11:21, among which directly attack scored 6 points (accounting for $47.6 \%$ of the total score). The Indonesian players send 5 points due to mistakes (accounting for $52.4 \%$ of the total score). The Chinese players directly mistakes 12 points in the first game (accounting for $57.12 \%$ of the total losing score). The major mistakes: 1 jump smash (accounting for $4.76 \%$ of the total losing score), 4 smash before the net (accounting for $19.04 \%$ of the total losing score), 6 pushing backcourt ball (accounting for $28.56 \%$ of the total losing score), 1 smash ball (accounting for $4.76 \%$ of the total losing score). Indonesian players score 9 points by direct attack (accounting for $42.84 \%$ of Chinese players' total losing points). Main factors of successful attack by Indonesian players: 2 serve (accounting for $9.52 \%$ of Chinese players' total losing points), 3 jump smash (accounting for $14.28 \%$ of Chinese players' total losing points), 3 pushing backcourt ball (accounting for $14.28 \%$ of Chinese players' total losing points), 1 smash (accounting for $4.76 \%$ of Chinese players' total losing points). (Table 1).

\section{The Serve Landing Area Change}

The serve landing area schematic diagram in badminton dou- bles is shown in Figure 1. Doubles serve can be divided into the frontcourt ball and backcourt ball. Moreover the frontcourt ball can be divided into Zone 1 and Zone 2 according to the landing area; the backcourt ball is divided into Zone 3, Zone 4.

The serve technique is one important technique in the doubles event; its impact on the outcome is far greater than the singles event. The quality of serve has a direct impact on active and passive, is an important link of score and mistakes. Serve accuracy and serve change rate are two reliable indicators that reflect the serve quality. We can see from Table 2 that the serve landing area of the top players in the world today are mostly in Zone 1 frontcourt, accounting for $83.7 \%$ of the total; followed by Zone 4 in the backcourt accounting for $7.2 \%$ of the total; the error rate of serve was $4.1 \%$; serve direct scoring rate was $6.6 \%$.

Through study of Table 2, we find that the serve success rate and stability of Sino-Indian players are much higher in the mixed doubles. Due to judgment errors, the error rate of Chinese players is slightly higher than that of the Indonesian players. We can see from the video data that there is no significant difference between He Hanbin/Yu Yang's serve landing area changes and that of Nova/Nasir. This is different with the research results of Chinese scholar Yongmin Cheng's article "Winning rule of badminton's double and the key of the competition ability of men's double”. His article thinks: serve change rate of Chinese athletes has a large gap with the world 
Table 2.

Serve landing zone and serve directly score table of each team.

\begin{tabular}{lccccccc}
\hline \multirow{2}{*}{ Serve athletes } & \multicolumn{3}{c}{ Serve landing zone } & \multicolumn{2}{c}{ Scores } & \multirow{2}{*}{ Total } \\
\cline { 2 - 6 } & Zone 1 & Zone 2 & Zone 3 & Zone 4 & Mistakes & & \\
\hline He Hanbin/Yu Yang (China) & $74(88.4 \%)$ & $4(4.8 \%)$ & $1(1.2 \%)$ & $2(2.4 \%)$ & $3(3.6 \%)$ & $4(4.8 \%)$ & 84 \\
Nova/Nasir (Indonesia) & $90(93.75 \%)$ & $2(2.08 \%)$ & $1(1.04 \%)$ & $1(1.04 \%)$ & $2(2.08 \%)$ & $6(6.25 \%)$ & 96 \\
Clark/Blair (England) & $13(59.1 \%)$ & $5(22.7 \%)$ & $1(4.5 \%)$ & $1(4.5 \%)$ & $2(9.1 \%)$ & $1(4.5 \%)$ & 22 \\
Jung Jae Sung/Lee Yong-dae (South Korea) & $25(80.6 \%)$ & $2(6.5 \%)$ & $2(6.5 \%)$ & $1(3.2 \%)$ & $1(3.2 \%)$ & $0(0.0)$ & 31 \\
\hline
\end{tabular}

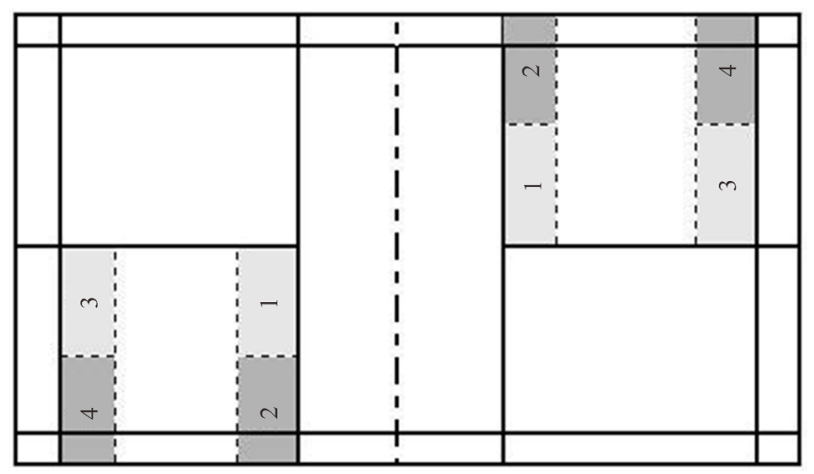

Figure 1.

The schematic diagram of badminton doubles venue.

top players; there are significant differences. This is perhaps due to in recent years we have made significant progress in the mixed doubles competition and training.

The effect of service and receiving has great relation with the serve quality. When the serve changes quickly and quality is high, the receiving side is relatively in a passive state. For serving side, it helps control the initiative of entire situation and has important significance on winning. Today's world-class mixed doubles player's service and receiving give priority initiative. This may be limited by the rules. Badminton serve is not much of a threat, which also verifies that it is not very easy to scoring by serve. One can only attain the goal to contain opponents through serve time change and placement change (Table 2).

\section{Technique Analysis of Winning and Losing Points by Jump Smashes}

Through the study of Table 3, Chinese players are significantly inferior to the Indonesian players in the jump smash technique. It indicates that Chinese player's ball control technique before the net is not in place, requiring the improvement of the technical level. The jump smash technique is mainly used in situations where due to the other side's mistakes the serve ball is too high, then directly smash on the ball. In the high level competition it doesn't appear too often but sometimes it is also a means to win.

\section{Technique Analysis of Winning and Losing Points by Net Lob}

By the research of data in Table 4, the net lob technique needs further improvement in the badminton mixed doubles. In the badminton mixed doubles match, the players of both sides are competition combination through year's coordination, and have good tacit understanding during the game. Moreover it is difficult to find neutral in front and back field during a high
Table 3.

Winning and losing points table of Sino-India athlete’s jump smash.

\begin{tabular}{|c|c|c|c|c|}
\hline \multirow{2}{*}{ Serve athletes } & \multicolumn{2}{|c|}{ Jump smash } & \multirow{2}{*}{ Numbers } & \multirow{2}{*}{ Scores } \\
\hline & Success & Mistake & & \\
\hline $\begin{array}{c}\text { He Hanbin/Yu Yang } \\
\text { (China) }\end{array}$ & 5 & 3 & 8 & 5 \\
\hline $\begin{array}{l}\text { Nova/Nasir } \\
\text { (Indonesia) }\end{array}$ & 7 & 0 & 7 & 7 \\
\hline
\end{tabular}

Table 4.

Winning and losing points table of Sino-India athlete's net lob.

\begin{tabular}{|c|c|c|c|c|}
\hline \multirow{2}{*}{ Serve athletes } & \multicolumn{2}{|c|}{ Net lob } & \multirow{2}{*}{ Numbers } & \multirow{2}{*}{ Scores } \\
\hline & Success & Mistake & & \\
\hline $\begin{array}{l}\text { He Hanbin/Yu Yang } \\
\text { (China) }\end{array}$ & 1 & 7 & 8 & 1 \\
\hline $\begin{array}{l}\text { Nova/Nasir } \\
\text { (Indonesia) }\end{array}$ & 3 & 7 & 10 & 3 \\
\hline
\end{tabular}

level competition. The only way is to violently smash to make the other side have mistakes. So the proceeding of net lob technique is prone to cause mistakes when the opponent is not in a proper place catching the ball.

\section{Technique Analysis of Winning and Losing Points by Pushing Backcourt Ball}

Through Table 5, we find that pushing backcourt ball is a technical method difficult to master like the above net lob in the badminton mixed doubles match. The success of pushing backcourt ball lies in two aspects: one is to seize the opportunity to push the ball to the opponent vacancy and score, the other is scoring by opponent's misjudgment. Pushing backcourt ball is a usual technical method in mixed doubles match. Through pushing backcourt ball, opponents can make mistakes in returning the ball. Then one can seize the opportunity to score. But improper master of the ball's intensity and direction can easily make the ball fly out of the field or the sideline. The pushing backcourt ball technique is not only a technical means causing opponent's mistake but also an important factor causing one's own mistake.

\section{Technique Analysis of Winning and Losing Points by Smash}

The smash technique is the most important score means in badminton game. If the ball is fast, it can easily and directly cause opponent's untimely response then score. Successful smash can also increase the mood and excitement of themselves and their teammates, making them more spirited in the game. Through the study of Table 6, although the scores by directly 
Table 5.

Winning and losing points table of Sino-India athlete's pushing backcourt ball.

\begin{tabular}{ccccc}
\hline \multirow{2}{*}{ Serve athletes } & \multicolumn{2}{c}{ Pushing backcourt ball } & Numbers & Scores \\
\cline { 2 - 3 } & Success & Mistake & & \\
\hline $\begin{array}{c}\text { He Hanbin/Yu Yang } \\
\text { (China) }\end{array}$ & 5 & 14 & 19 & 5 \\
$\begin{array}{c}\text { Nova/Nasir } \\
\text { (Indonesia) }\end{array}$ & 11 & 14 & 25 & 11 \\
\hline
\end{tabular}

Table 6.

Winning and losing points table of Sino-India athlete’s smash.

\begin{tabular}{|c|c|c|c|c|}
\hline \multirow{2}{*}{ Serve athletes } & \multicolumn{2}{|c|}{ Smash } & \multirow{2}{*}{ Numbers } & \multirow{2}{*}{ Scores } \\
\hline & Success & Mistake & & \\
\hline $\begin{array}{l}\text { He Hanbin/Yu Yang } \\
\text { (China) }\end{array}$ & 6 & 4 & 10 & 6 \\
\hline $\begin{array}{l}\text { Nova/Nasir } \\
\text { (Indonesia) }\end{array}$ & 7 & 2 & 9 & 7 \\
\hline
\end{tabular}

smash is not high, smash technique is the most widely used technical means in the badminton competition. During doubles matches, both sides play with a tacit understanding. One cannot directly kill the ball in several shoots, so the number of success is not huge.

\section{Conclusion}

This paper analyzes the main scoring techniques: the serve technique, the jump smash technique, the net lob technique, the pushing backcourt ball technique and the smash technique by watching the game video of the 29th Olympic Games by He Hanbin/Yu Yang (China) and Nova/Nasir (Indonesia). The analysis shows that: in the badminton mixed doubles match, since both sides are partners for many years, in order to win the game you must have good badminton technique; particularly the error rate of the net lob technique and the pushing backcourt ball technique is high, which needs further practice.

In face of pressure, China's two young athletes seem lack of experience. In the face of the sophisticated Indonesian opponents Nasir/Nova, they adhere to the final, but failed. Although the result of the game is not satisfactory, but the whole process illustrates that the young combination will be the next Olympic legend. In last year's Danish Open badminton, He Hanbin/Yu Yang won the Super Series champion first time after the pairing. After the match IBF (International Badminton Federation) official website expressed great interest in this "Star of Hope", and hailed them as new hope of Chinese mixed doubles.

The semi-final opponents Nasir/Nova are the strongest mixed doubles players in the world following Zhang Jun/Gao Ling. In the 2005 and 2007 World Championships, Nasir/Nova won the champion twice. The two not only have tacit cooperation, but also Nasir's net technology and Nova's key ball handling are both superior; meanwhile they have rich experience in big competition, and they are the strongest opponents for Chinese mixed doubles at the Olympics. In the record against them, the Chinese team Zheng Bo/Gao Ling have advantages, while He Hanbin/Yu Yang are obvious in disadvantage (Chen, 2009).

In the match two young athletes obviously lack some preparation. Especially Yu Yang has a marked change psychologically after winning the doubles champion; she always feels a little nervous about the outcome on the court. He Hanbin despite has the brave killing method, but the young edgy defects result in his mistakes constantly. This is particularly evident in the deciding game. Although He Hanbin/Yu Yang have great energy, but the handling of key balls still seems immature. At first they should have grasped the field point several times, and won in one fell swoop; but they repeatedly have mistakes leading to the final defeat.

Objectively speaking, He Hanbin/Yu Yang have no big shortcoming in technique. The biggest shortcoming is lack of experience, especially the experience of the Olympic Games. Badminton is not only a game of physical and technical; more often psychology can decide the outcome of the game. Young athletes are not very "seasoned" in this aspect especially. However, after the baptism of various big and small competitions, they will perhaps become the hope of the future of Chinese badminton mixed doubles combination.

\section{REFERENCES}

Chen, Y. (2009). Strategies and tactics on the badminton competition. Badminton, 6, 56-57.

Cheng, Y. M. (2005). Effect of skill style on performance of men's double of China badminton team. Journal of Shandong Physical Education Institute, 21, 88-90.

Cheng, Y. M. (2008). Winning rule of badminton's double and the key of the competition ability of men's double. Journal of Shandong Physical Education Institute, 24, 83-85.

Geng, Y. D. (2004). Analysis of selective principle and implement outline for badminton tactics. Journal of Shaoguan University, 25, 9395.

Pan, L. (2001). The influence of new game system on badminton doubles of lady competitors. Journal of Nanjing Institute of Physical Education, 15, 138-139.

Tian, J. N. (2004). Study on third strike of serving side to force the pace in men's double event of badminton. Journal of Nanjing Institute of Physical Education (Natural Science), 12, 37-39.

Zhang, B., \& Feng, Y. (2013). The special quality evaluation of the triple jump and the differential equation model of long jump mechanics based on gray correlation analysis. International Journal of Applied Mathematics and Statistics, 40, 136-143. 